\title{
Sharp edge preservation using bicubic B-spline surfaces
}

\author{
Khang Jie Liew*, Ahmad Ramli, Nur Nadiah Abd Hamid, Ahmad Abd Majid \\ School of Mathematical Sciences, Universiti Sains Malaysia, 11800 Minden, Penang \\ *Corresponding author, e-mail: kenji_liewkj@yahoo.com.my
}

Received 1 Dec 2014

Accepted 21 May 2017

\begin{abstract}
This paper investigates the sharp edge preservation of B-spline surfaces. A $K$-nearest neighbour search incorporating an averaging method is proposed to detect a sharp edge from a set of data points. In this study, the unknown B-spline control points are determined, followed by the fitting of the sample of data points using a bicubic B-spline surface. The sample of data points within the sharp edge area are initially marked by the bootstrap method. Next, a particular set of control points is repeated, while the set of data points is projected onto the constructed bicubic B-spline surface for the purpose of preserving the sharp edge. The present study is also determines the effect of noise with different noise levels in preserving the sharp edge. The experimental results demonstrate that the sharp edge in sample data points is well-preserved using the proposed method which is slightly influenced by the presence of noise.
\end{abstract}

KEYWORDS: averaging method, $K$-nearest neighbour search method, noisy data, surface reconstruction

\section{INTRODUCTION}

Nowadays, the advancement of scanning technologies allows for better accuracy to be achieved by providing a dense set of data points during the scanning process from the real life model. However, some of the critical parts of the model are unable to be recovered due to the low number of control points during the surface reconstruction process. Moreover, the critical parts tend to occur at the sharp edge, thus complicating the recovering process. The sharp edge can be defined as an edge or line between two surfaces or a corner which meets the surfaces. In relation to this, it will be very useful to identify and detect the particular point sets, that belong to the sharp edge for the purpose of recovering the sharp edges.

There are two techniques that can be applied to extract the sharp edges, namely, polygonal meshes and point-based method. However, the lack of information of normal and connectivity of the point set models has caused the feature detection on point sets to be difficult compared to the detection on meshes. However, it is the clear that the aim of this study is to work directly on the sample of point set instead of the polygonal meshes because the scanning devices are able to generate a set of data points that resemble the original surface instead of mesh.
Hence it is necessary to investigate the method used for feature detection and its preservation.

The detection of sharp features on point sets is not only crucial to fulfil the quality measurement, but it is also equally important for reverse engineering with mesh generation, surface reconstruction, simplification, or segmentation that requires the sharp edges to be preserved ${ }^{1}$. There are also moving least square (MLS) surface reconstruction methods that seek for sharp edge reconstruction that is commonly used for noisy data points set. However, additional steps are required to be taken because smooth surfaces are generally produced. Meanwhile, other related works on edge detection can be seen in Ref. 1. The proposed Gauss map clustering is believed to be able to overcome the shortcoming of MLS method. The normal and connectivity information are not provided, but the detection of sharp edges is fully automatic and the algorithm works directly to mark the sharp features of the point cloud.

The statistical method which is known as the variance-based feature detection was used ${ }^{2}$. The variance values are obtained from the normal estimation conducted on a model, which is then calculated based on the principal component analysis and further resampling is performed using the bootstrap method. The variance values will be able to provide 
an indication on the surface with feature or sharp edges. It is worth to note that a high variance value is caused either by the noise or the presence of a sharp feature. Hence a high variance value indicates area with sharp edges by assuming that the noise level is uniform. However, the limitation for this method is described based on the noise level, where a high noise level will cause the variance value for the non-feature area to be high.

In this paper, an algorithm that utilizes the $K$ nearest neighbour search by incorporating the averaging method is proposed in order to fit the sample of data points using the bicubic B-spline surface. In relation to this, the proposed algorithm tends to focus on the sharp edge preservation instead of the surface approximation or interpolation. The work conducted for the purpose of detecting the sharp edge using variance-based feature detection method is further extended and integrated in this study ${ }^{2}$. The variance values were determined from the bootstrap method, which is expected to assist in marking the part where the feature takes place by assuming that the sample of data points is uniformly distributed.

A number of aspects will be briefly introduced which are the mathematical background of B-spline surface, $K$-nearest neighbour search method, and the continuity. The method applied to fit the sample data points is the proposed algorithm, while the process of preserving shape edge will be described in the section of materials and methods. Meanwhile the graphical results of the sharp edge preservation via bicubic B-spline surface performed using the proposed algorithm is presented in the results section. Moreover, the effect of noise with different noise levels towards the sharp edge preservation is also shown graphically. Next, the sharp edge preservation and several other issues in this study will be reviewed. On top of that, the effect of noise on the surface sharp edge preservation will also be further discussed, and finally, a conclusion will be provided at the end of this paper.

\section{MATERIALS AND METHODS}

In this paper, a set of sample data points is taken from the point cloud of cube together with their respective variance values that act as an input. Meanwhile, a tensor product of bicubic B-spline surfaces with the preserved sharp edge is produced as an output. In the context of this study, an algorithm with the combination $K$-nearest neighbour search and averaging method is proposed to fit a set of 3D data points using B-spline surface. The main objective of this study is to recover and preserve the sharp edge of the bicubic B-spline surface. In relation to this, sharp edge can be defined as an edge or line between two surfaces or a corner, provided that the surfaces meet together. Furthermore, some of the related mathematical background will be provided in the following sections.

\section{B-spline surface}

The rectangular B-spline patch surface $f(u, v)$ is constructed by applying tensor product technique to the $\mathrm{B}$-spline curve, which is described as a linear combination of B-spline basis functions in two topological parameter ${ }^{3} u$ and $v$. Furthermore, B-spline surface is defined by a topological rectangular set of control points $P_{i, j}$ for $0 \leqslant i \leqslant m, 0 \leqslant j \leqslant n$ as well as the two knot vectors $U=\left\{u_{0}, u_{1}, u_{2}, \ldots, u_{m+k}\right\}$ and $V=\left\{v_{0}, v_{1}, v_{2}, \ldots, v_{n+l}\right\}$, whereby the B-spline surface patch is given by

$$
f(u, v)=\sum_{i=0}^{m} \sum_{j=0}^{n} P_{i, j} N_{i}^{k}(u) N_{j}^{l}(v),
$$

where $N_{i}^{k}(u)$ and $N_{j}^{l}(v)$ serve as the B-spline basis functions of orders $k$ (degree $k-1$ ) and $l$ (degree $l-1$ ), respectively. The parameters $u$ and $v$ are described as the global parameters. In this study, Bspline surface of degree $3(k=l=4)$ is used. This surface is called bicubic B-spline surface.

\section{$K$-nearest neighbours algorithm}

The $K$-nearest neighbours (KNN) algorithm is one of the simplest machine learning algorithms. In this case, the $K$ is a positive integer. This particular algorithm specifically seeks for $K$ points that are relatively close to one point considered from a set of points in $m$-dimensional space. The metric used in measuring the closeness known as the Euclidean distance, in which the distance between two points $X=\left(x_{1}, x_{2}, \ldots, x_{m}\right)$ and $Y=\left(y_{1}, y_{2}, \ldots, y_{m}\right)$ is $^{4}$

$$
d(X, Y)=\sqrt{\sum_{i=1}^{m}\left(x_{i}-y_{i}\right)^{2}} .
$$

$K$ is a user-defined value, thus it has to be chosen carefully. Moreover, the noise will be modelled if the $K$ value is too small, whereas many points from other classes may be included by the neighbours in the case of a large $K$. This algorithm is very simple with no training involved, but it is costly because it requires the same searching procedure which to be repeated for every single point in a point set. 


\section{Bootstrap method}

The bootstrap method is a statistical method that is conducted based on the repetition of random resampling of the data as well as the average of the results obtained from each sample. The reuse of the data is the result of repetitive resampling which is considered helpful when the available data are sparse. The following descriptions are related to the bootstrap method ${ }^{5}$.

Assume that a set of sample data is comprised of of $N$ data points or training points, whereby $V=$ $\left\{v_{1}, v_{2}, \ldots, v_{N}\right\}$. In this case, each element in set $V$ which is labelled as $v_{i}$ is in the form of 3D coordinates, represented as $v_{i}=\left(x_{i}, y_{i}, z_{i}\right)$. The bootstrap set is produced through a random sampling of the $N$ elements from $V$ with replacement. Meanwhile, the expected number of distinct elements in bootstrap set $V^{* b}$ is supposed to be lower than $V$ based on the random resampling performed with replacement. The sampling procedure is repeated $B$ times in order to produce $B$ independent bootstrap sets, $V^{* b}$ where $b=1,2,3, \ldots, B$.

Suppose that $S(V)$ is represented by any quantity computed from the data $V$; hence, the bootstrap samples, $V^{* b}$ can be used to estimate any aspect of the distribution of $S(V)$. For instance, the mean can be estimated based on the bootstrap mean as

$$
\bar{S}(V)=\frac{1}{B} \sum_{b=1}^{B} S\left(V^{* b}\right),
$$

while the variance can be estimated by the bootstrap variance as

$$
\hat{S}(V)=\frac{1}{B-1} \sum_{b=1}^{B}\left(S\left(V^{* b}\right)-\bar{S}(V)\right)^{2} .
$$

Next, consider a set of data points with size $N$, represented as $D=\left\{d_{1}, d_{2}, \ldots, d_{N}\right\}$. The $K$-nearest neighbourhood of $d_{i} \in D$ for $i=1,2, \ldots, N$ is defined as $\mathscr{N}_{i}=\left\{p_{1}, p_{2}, \ldots, p_{K}\right\}$, where $p_{j} \in D$ for $j=$ $1,2, \ldots, K$. The bootstrap variance for $d_{i}$, denoted as $\hat{S}\left(\mathscr{N}_{i}\right)$, can be determined using (4).

\section{Continuity}

The concern of this subsection revolves around the continuity that occurs at the joint of two neighbouring curve segments. Hence it is extremely important to know how the individual segments can be connected. There are two types of continuity to be considered, namely, parametric continuity and geometric continuity ${ }^{6}$.
Parametric continuity, $C^{n}$, is known as $n$ th-order parametric continuity, where $n$ is usually represented by 0,1 , and 2 . The algebra is used to describe the smoothness of the parameters' value along the curve.

Geometric continuity, $G^{n}$, is known as $n$ th-order geometric continuity, where $n$ is normally represented by 0,1 , and 2 . Geometric continuity is considered as a less restrictive than the parametric continuity. Furthermore, the curve is expected to have $C^{0}$ continuity which also implies $G^{0}$ continuity if the two consecutive segments meet at a point. The curve may have $G^{1}$ continuity if the directions of the tangent vectors of the two segments are similar at the join point. In general, a curve has $G^{n}$ continuity at a join point, provided that every pair of the first $n$ derivatives of the two segments are in the same direction at the join point. The $G^{0}, G^{1}$, and $G^{2}$ continuity are also referred to the point, tangent, and curvature continuity, respectively.

Moreover, the curve is said to have $C^{n}$ if the same derivatives also have identical magnitudes at the join point. A curve that has $C^{n}$ continuity at a join point implies $G^{n}$ continuity, considering that $C^{n}$ is more restrictive than $G^{n}$, but the inverse will not necessarily be true. Finally, a curve with continuous tangent and curvature vectors is said to have $G^{2}$ continuity.

In general, B-spline surface in (1) with multiplicity $\alpha$ will have $C^{(k-\alpha-1)}$ and $C^{(l-\alpha-1)}$ continuities in the direction of $u$ and $v$, respectively. Multiplicity is the number of knots or control points being repeated consecutively ${ }^{7,8}$. When a control point is repeated, that is the multiplicity increases, the resulting surface moves closer to that point ${ }^{7}$. For the case of bicubic B-spline surface, the surface is forced to interpolate that control point resulting a surface with $C^{0}$ continuity if the control point is given a multiplicity of $3^{7}$.

\section{Preserving the sharp edge on surface}

The previous work on the variance-based method conducted for the purpose of detecting the sharp edge from the set of points is extended with the focus of recovering the sharp edge during the surface reconstruction. In this section, the variance values are obtained by determining it from the bootstrap method performed earlier. These variance values are helpful in marking the part where the sharp edge occurs, with the assumption that the sample of data points is uniformly distributed. A particular row or column of control points is repeated to achieve the sharp edge preservation. Hence a particular row 
of control points will be repeated 3 times for the bicubic B-spline surface. The following example is provided to enhance the understanding.

Example 1 Given a set of control points

$$
\begin{aligned}
&\left\{\left\{\mathbf{r}_{11}, \mathbf{r}_{12}, \mathbf{r}_{13}, \mathbf{r}_{14}\right\},\left\{\mathbf{r}_{21}, \mathbf{r}_{22}, \mathbf{r}_{23}, \mathbf{r}_{24}\right\}\right. \\
&\left\{\mathbf{r}_{31}, \mathbf{r}_{32}, \mathbf{r}_{33}, \mathbf{r}_{34}\right\},\left\{\mathbf{r}_{41}, \mathbf{r}_{42}, \mathbf{r}_{43}, \mathbf{r}_{44}\right\} \\
&\left.\left\{\mathbf{r}_{51}, \mathbf{r}_{52}, \mathbf{r}_{53}, \mathbf{r}_{54}\right\}\right\}
\end{aligned}
$$

where $\mathbf{r}_{i j} \equiv\left(x_{i j}, y_{i j}, z_{i j}\right)$, we assume that the third row control points are $\left\{\mathbf{r}_{31}, \mathbf{r}_{32}, \mathbf{r}_{33}, \mathbf{r}_{34}\right\}$, and suppose that they are detected as the part of sharp edge. Hence the particular row is required to be repeated three times in order to preserve the sharp edge for a bicubic B-spline surface. The result of the process is

$$
\begin{aligned}
& \left\{\left\{\mathbf{r}_{11}, \mathbf{r}_{12}, \mathbf{r}_{13}, \mathbf{r}_{14}\right\},\left\{\mathbf{r}_{21}, \mathbf{r}_{22}, \mathbf{r}_{23}, \mathbf{r}_{24}\right\},\right. \\
& \left\{\mathbf{r}_{31}, \mathbf{r}_{32}, \mathbf{r}_{33}, \mathbf{r}_{34}\right\},\left\{\mathbf{r}_{31}, \mathbf{r}_{32}, \mathbf{r}_{33}, \mathbf{r}_{34}\right\} \text {, } \\
& \left\{\mathbf{r}_{31}, \mathbf{r}_{32}, \mathbf{r}_{33}, \mathbf{r}_{34}\right\},\left\{\mathbf{r}_{41}, \mathbf{r}_{42}, \mathbf{r}_{43}, \mathbf{r}_{44}\right\} \text {, } \\
& \left.\left\{\mathbf{r}_{51}, \mathbf{r}_{52}, \mathbf{r}_{53}, \mathbf{r}_{54}\right\}\right\} .
\end{aligned}
$$

This means that the level of continuity at the repeated part is decreased from $C^{2}$ to $C^{0}$, which implies $G^{2}$ to $G^{0}$. The proposed algorithm is as follows.

Algorithm 1 Sharp edge preservation on bicubic B-spline surface. Input: sample data points $P=$ $\left\{\left(x_{\mathrm{c}}, y_{\mathrm{c}}, z_{\mathrm{c}}\right)\right\}$. Output: Bicubic B-spline surface with sharp edge.

Step 1: Obtain the variance from the bootstrap method for each sample data points using (4).

Step 2: Determine the positive standardized variance values, denoted as $w_{i}$.

Step 3: Use the rotation matrix $M$ to reorient the data points ${ }^{9}$.

Step 4: Set coordinates $p_{z}=0$ for the initial set of control points, $\phi_{i j}=\left(p_{x}, p_{y}, p_{z}\right)$.

Step 5: Update the $p_{z}$ value by taking the value for the sum of product of $z$-coordinate of the 2-nearest data points, $z_{i}$, with its positive respective standardized variance values, $w_{i}$, and divided by its respective sum of positive standardized variance values, $w_{i}$, which can be formulated as $\sum_{i=1}^{2} z_{i} w_{i} / \sum_{i=1}^{2} w_{i}$. This iterative process is repeated three times.

Step 6: Reorient the sample data points as well as the updated set of control points to its original position through the inverse of rotation matrix, $M^{-1}$.
Step 7: Calculate the average value of the standardized variances of each control points, $v$ with respect to the 4-nearest data points. If $v>e$, where $e$ is a user-defined (in this case is 0.20), then mark the particular control point as 1 to identify it as a sharp edge, while 0 as the nonsharp edge.

Step 8: Transpose the set of control points if it is in the form of vertical sharp edge.

Step 9: Calculate the sum of the marking values for a row of control points, $S$. If $S>g$, where $g$ is a user-defined (in this case is 4 ), then repeat the row of control points for three times.

Step 10: Plot the bicubic B-spline surface patch based on the obtained control points.

Step 11: Project the sample data points $P=$ $\left\{\left(x_{c}, y_{c}, z_{c}\right)\right\}$ onto the constructed surface.

According to Algorithm 1, $w_{i}$ in Step 5 serves as weight for the $z_{i}$ in the formula. The variance values obtained from the bootstrap method is necessary in order to standardize and project the value to be positive before the variance value is used. The standardization is carried out as a result of some variance values being equal to zero. The variance values will have a negative or positive value after the standardizing process. Meanwhile, the mean and the standard deviation for the standardized variance values will be 0 and 1 , respectively. Next, all the standardized variance values are projected to the positive values for the purpose of preventing the effect of negative standardized variance values on the surface fitting. The iterative process in Step 5 is repeated for three times to ensure that the value of $p_{z}$ will be stable eventually.

It is well aware that the small value of $K$ is chosen in performing the $K$-nearest neighbour search method in Step 5 and 7 considering that the distance within the points is near to each other. In Step 9, the user-defined value is 4 due to the presence of 6 control points in a row for this study. However, the projection procedure in Step 11 is an optional step. The smoothing effect can be achieved after finding the $z$-coordinate on the bicubic B-spline surface with respect to the $x_{\mathrm{c}}$ and $y_{\mathrm{c}}$.

\section{Effects of noisy data on sharp edge preservation}

In this subsection, the effect of noisy data on the sharp edge preservation will be analysed. In this context, the noise is defined as the variation in a set of data points. The presence of noise does not only result in the bad fitting of the surface, but may also smooth up certain parts of the sharp 
edge. Moreover, the accuracy of the 3D model will be reduced during the surface reconstruction due to the set of data points being contaminated by noise. Nevertheless, it is not the exactly the case when many of the surface reconstruction procedures tend to assume that the distribution of the noise is Gaussian or normal distribution ${ }^{10}$.

A sample of data points assumed to be noisefree is collected to carry out this experiment. Next, Algorithm 1 is applied in the experiment, where 13 noisy data points are randomly added in positive and negative vertical direction for the noise-free data points. The $d$-noise level implies a simulated noise which is added through normal distribution with the variance of $h d$, where the $h$ is referred as the average distance between the two nearest points from a set of points. The noise levels $d$ to be considered include $0.16,0.32,0.48$, and 0.64 . The $h$ value which serves as the average distance of two nearest points of this study is 0.03125 . Hence the respective noise values, $h d$, are $0.005,0.010,0.015$, and 0.020 . Finally, the effect of noisy data is visually inspected by comparing the noise-free sample with the noise-added sample.

\section{RESULTS AND DISCUSSION}

An easy method that utilizes the $K$-nearest neighbour search and incorporates the averaging method is proposed to determine the set of control points. The main purpose of this paper is to focus on sharp edge preservation, thus the B-spline surface fitting is excluded in this study. Next, the number of the control points also needs to be considered because it will have an effect on the sharp edges of the B-spline surface. In this study, the dimension of the control points net is set specifically to $6 \times 6$ as a result of the distribution of the data points.

The model of this study is tested with an area from a cube point set model containing 12 sharp edges. A sample region with a sharp edge consisting 51 data points is chosen in order to test the performance of the algorithm on the data points. The results obtained from Algorithm 1 will be presented in this section. It is crucial to note that the sample data points are denoted as green dots, whereas the control points are denoted as red dots. The sample data point is shown in Fig. 1.

The bicubic B-spline surfaces in Fig. 2a,b have $C^{2}$ continuity. The reorientation is conducted to facilitate the determination of a set of control points (Fig. 2). The effect of noisy data on the sample data points assumed to be noise-free are observed. Meanwhile, the noise-free surface can be seen in

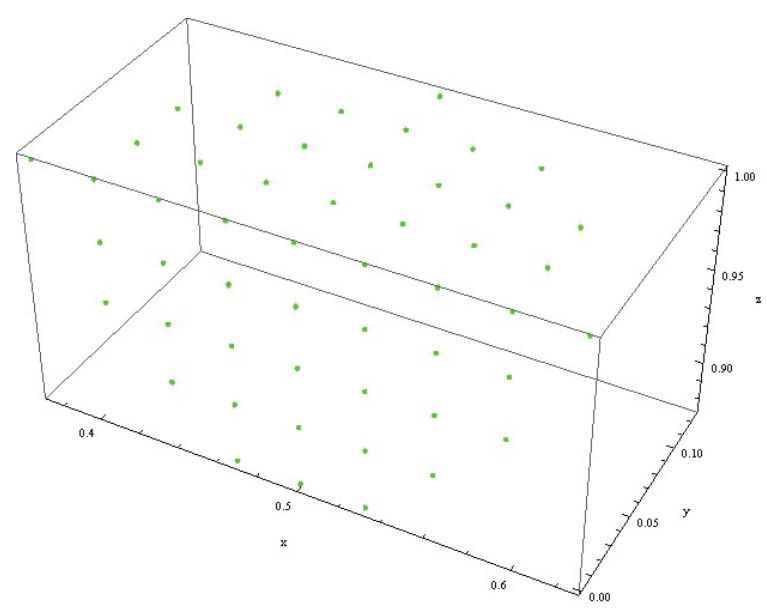

Fig. 1 A sample of 51 data points from the cube point set model.
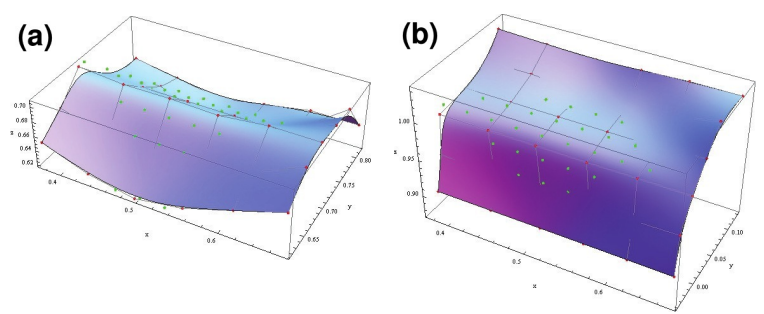

(c)
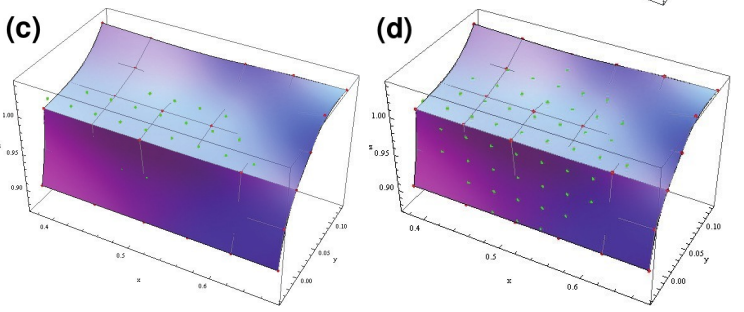

(d)

Fig. 2 The process of sharp edge preservation according to Algorithm 1. The sample of data points is (a) reoriented and an initial bicubic B-spline surface is constructed, (b) reoriented to its original position. The final bicubic Bspline surface with sharp edge (c) before projection and (d) after projection.

Fig. 2. The 13 noise data is randomly added to the sample of noise-free data points in positive and negative vertical direction. Next, the effect of noisy data on sharp edge preservation is visually inspected. The noise level of $0.16,0.32,0.48$, and 0.64 are chosen for experimental purpose. The observations for the bicubic B-spline surface with sharp edge (after projection) are shown in Fig. 3.

According to Fig. 3, a slight bump at the sharp edge part is observed when the noise level is increased from $0.16-0.64$, but it is still considered well preserved. However, the sharp edge part is un- 

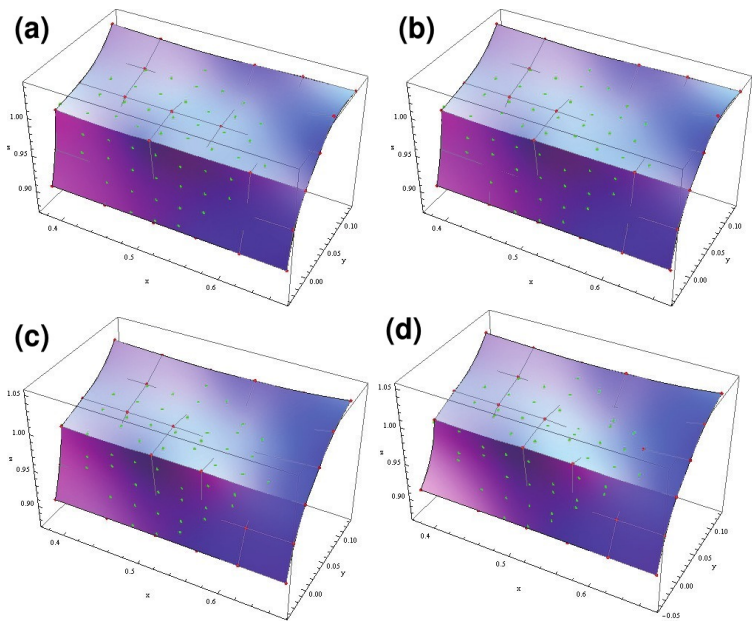

Fig. 3 The effect of noise towards sharp edge preservation on the final bicubic B-spline surface with sharp edge with different noise levels; (a) 0.16, (b) 0.32, (c) 0.48, (d) 0.64 .

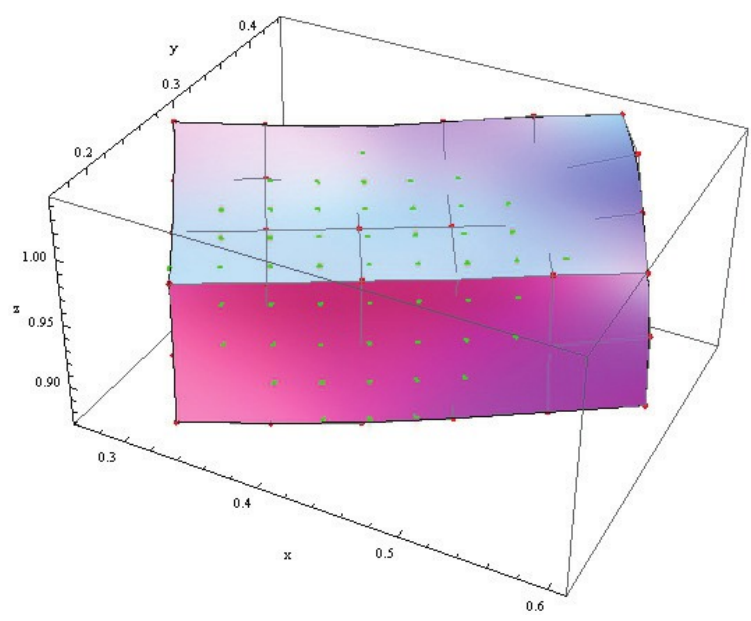

Fig. 4 The bicubic B-spline surface where the sharp edge is not in the direction of $x, y$, or $z$-axis.

able to be recovered in the higher noise level. Apart from that, the reorientation of the data points along the correct main axis is necessary to be performed if the sharp edge does not occur in the direction of $x, y$, or $z$-axis. Algorithm 1 can be applied after the reorientation is performed. The surface then needs to be reoriented to its original position following the orientation. Nevertheless, the sharp edge cannot be recovered without a proper reorientation using this approach; hence it will remain smooth after the surface reconstruction. Fig. 4 is an example of the sharp edge region which is not placed along any of the three main axes, but the sharp edge turns out to be well preserved after the reorientation. In this case, the orientation is performed manually.

In short, the certain row of control points is repeated $k-1$ times to preserve the sharp edge, thus showing that the sharp edge part has $C^{0}$ and $G^{0}$ continuity. The process of repeating the $k-1$ knots is an alternative method that is applied to preserve the sharp edge, even though the sharp edge is known to have both $C^{0}$ and $G^{0}$ continuity. However, the process of detecting which knots to be repeated has become difficult. Another issue that needs to be taken into account is the fitting problem, in which it can only be well-fitted if the data points are sufficient; otherwise it will cause the corners of the surface patch to be curvy.

\section{CONCLUSIONS}

This paper describes and discusses the sharp edge preservation in bicubic B-spline surface. In relation to this, the sharp edge preservation in bicubic Bspline surface was also observed. According to the result obtained from the sharp edge detection method known as the variance-based feature detection method, it was discovered that each control point managed to be marked by applying the $K$ nearest neighbour search method. The particular set of control points was repeated for three times to ensure the sharp edge is achieved. The result showed that the proposed algorithm is capable in preserving the sharp edge. Moreover, the effect of noisy data on sharp edge preservation will slightly influence the sharp edge area and the smoothness of the bicubic B-spline surface. In regard to this, there is a possibility that the sharp edge will not be able to recover if a high level of noisy data is present, which will eventually smooth up the sharp edge area. Hence it is safe to conclude that the denoising process is important to be carried out as the pre-process of the surface reconstruction.

Acknowledgements: The authors would like to thank the referees for their comments and suggestions that have helped to improve this manuscript. The authors are extremely thankful and would also like to extend their gratitude to the School of Mathematical Sciences, Universiti Sains Malaysia for financial support provided by USM short terms grant (No. 304/PMATHS/6312136) and RUI grant (No. 1001/PMATHS/8011014). Finally, the main author of this manuscript also gratefully acknowledges the generous financial support contributed by the Ministry of Higher Education Malaysia under MyPhD scholarship. 


\section{REFERENCES}

1. Weber C, Hahmann S, Hagen H (2010) Sharp feature detection in point clouds. In: Proceedings of IEEE International Conference on Shape Modeling and Applications (SMI 2010), pp 175-86.

2. Ramli A, Ivrissimtzis I (2010) Bootstrap-based normal reconstruction. In: Proceedings of Curves and Surfaces 2010, Lecture Notes in Computer Science vol 6920, pp 575-85.

3. Cohen FS, Ibrahim W, Pintavirooj C (2000) Ordering and parameterizing scattered $3 \mathrm{D}$ data for B-spline surface approximation. IEEE Trans Pattern Anal Mach Intell 22, 642-8.

4. Han J, Kamber M (2001) Classification and prediction. In: Data Mining: Concepts and Techniques, Elsevier, pp 314-5.

5. Hastie T, Tibshirani R, Friedman J (2001) Model assessment and selection. In: The Elements of Statistical Learning: Data Mining, Inference, and Prediction, Springer, pp 249-50.

6. Salomon D (2011) The Computer Graphics Manual, Texts in Computer Science, Springer, pp 450-2.

7. Agoston MK (2005) Computer Graphics and Geometric Modeling, Springer-Verlag, pp 411-66.

8. Salomon D (2006) Curves and Surfaces for Computer Graphics, Springer Science \& Business Media, pp 263-5.

9. Farin G, Hansford D (2005) Practical Linear Algebra: A Geometry Toolbox, A K Peters, pp 199-221.

10. Sun X, Rosin PL, Martin RR, Langbein FC (2009) Noise analysis and synthesis for 3D laser depth scanners. Graph Model 71, 34-48. 